\title{
NUCLEUS: Exploring Coherent Neutrino-Nucleus Scattering with Cryogenic Detectors
}

\section{J. Rothe, et al. [full author details at the end of the article]}

Received: 29 July 2019 / Accepted: 24 November 2019 / Published online: 10 December 2019

(c) The Author(s) 2019

\begin{abstract}
The NUCLEUS experiment aims for the detection of coherent elastic neutrino-nucleus scattering at a nuclear power reactor with gram-scale, ultra-low-threshold cryogenic detectors. This technology leads to a miniaturization of neutrino detectors and allows to probe physics beyond the Standard Model of particle physics. A $0.5 \mathrm{~g}$ NUCLEUS prototype detector, operated above ground in 2017, reached an energy threshold for nuclear recoils of below $20 \mathrm{eV}$. This sensitivity is achieved with tungsten transition edge sensors which are operating at temperatures of $15 \mathrm{mK}$ and are mainly sensitive to non-thermal phonons. These small recoil energies become accessible for the first time with this technology, which allows collecting large-statistics neutrino event samples with a moderate detector mass. A first-phase cryogenic detector array with a total mass of $10 \mathrm{~g}$ enables a 5-sigma observation of coherent scattering within several weeks. We identified a suitable experimental site at the Chooz Nuclear Power Plant and performed muon and neutron background measurements there. The operation of a NUCLEUS cryogenic detector array at such a site requires highly efficient background suppression. NUCLEUS plans to use an innovative technique consisting of separate cryogenic anticoincidence detectors against surface backgrounds and penetrating (gamma, neutron) radiation. We present first results from prototypes of these veto detectors and their operation in coincidence with a NUCLEUS target detector.
\end{abstract}

Keywords Transition-edge sensor - Cryogenic detector · Coherent Neutrino-Nucleus Scattering

\section{CEvNS at a Nuclear Power Reactor}

Since its proposal in 1974 [1], coherent elastic neutrino-nucleus scattering (CEvNS ) has attracted growing attention in particle physics. It was first observed in 2017 by the COHERENT collaboration [2] using the neutrino emission of the Spallation Neutron Source (SNS) at the Oak Ridge National Laboratory. Acting as a stopped-pion source, 
SNS provides the largest neutrino energies (up to $53 \mathrm{MeV}$ ) participating in the coherent interaction and thus the largest interaction cross sections. An advantage of the stoppedpion source is its pulsed nature, which allows to greatly suppress backgrounds with a beam-on/beam-off comparison measurement. Recently, a number of projects are attempting an observation of CEvNS at a nuclear power reactor [3-9]. At a nuclear reactor, neutrinos arrive without a time tag; therefore, exquisite control of backgrounds is required. Reactor-off measurement periods, if available, are valuable ingredients in the modeling and understanding of backgrounds. With a typical reactor neutrino energy of around $3 \mathrm{MeV}$, the interaction cross section and observable nuclear recoil energies (which both scale with $E_{v}^{2}$ ) are around two orders of magnitude lower compared to a stopped-pion source. While the lower recoil energies have to be detected with a more sensitive apparatus, the available neutrino flux near a power reactor can more than overcompensate the lower cross section, allowing large-statistics experiments with a small target mass. Various scenarios of physics beyond the Standard Model can be probed in such an experiment (see [10-12] and references therein).

\section{The NUCLEUS Concept}

NUCLEUS is a reactor neutrino experiment aiming at an observation of CEvNS using a target consisting of cryogenic detectors. Its defining features are a demonstrated ultra-low-energy threshold for nuclear recoils as well as a sophisticated combination of cryogenic veto detectors designed to suppress external backgrounds. The experiment plans to deploy $\mathrm{CaWO}_{4}$ and $\mathrm{Al}_{2} \mathrm{O}_{3}$ detectors in the first physics phase NUCLEUS - $10 \mathrm{~g}$, transitioning to germanium and silicon detectors for the future upgrade NUCLEUS - $1 \mathrm{~kg}$. Initial tests are being performed with $\mathrm{Al}_{2} \mathrm{O}_{3}$ detectors, with other materials in preparation.

\subsection{Gram-Scale Cryogenic Calorimeters}

The NUCLEUS target detectors are evolved from technology used in the CRESST direct dark matter search experiment [13,14]. CRESST-III detectors [15] are $24 \mathrm{~g}$ $\mathrm{CaWO}_{4}$ crystals equipped with evaporated tungsten TES with a transition temperature tuned to $15 \mathrm{mK}$. By reducing the absorber size, the energy threshold can be improved. This leads to the idea of a "gram-scale cryogenic calorimeter" with ultralow thresholds [16]. In 2017 a first prototype, a $\mathrm{Al}_{2} \mathrm{O}_{3}$ cube of $5 \mathrm{~mm}$ side length, was operated above ground in Munich [17]. Due to its small size, the overall particle rate was small (around $0.1 \mathrm{~Hz}$ ), allowing the detector to operate stably. It achieved an energy threshold of $19.7 \mathrm{eV}$ for nuclear recoils and was used to place leading limits on spin-independent interactions of low-mass dark matter [18]. This sensitivity shows the readiness of this technology for a measurement of CEvNS. 


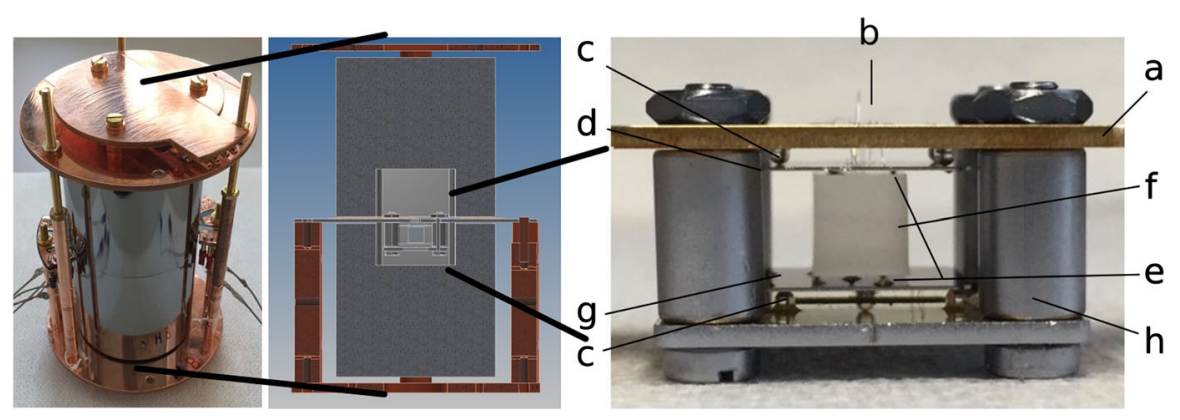

Fig. 1 The NUCLEUS 1g demonstrator. Left: photograph of complete prototype with outer vetos encapsulating the inner detectors. Center: cut view of the detector assembly including target, inner and outer vetos. Right: inner detectors. From top to bottom: thick silicon support wafer (a) with electrical and thermal contacts (b), sapphire balls (c) as spacers, top inner veto (d) with etched pyramids (e) and window for target contacts, target detector (f), bottom inner veto $(\mathrm{g})$. The tension of the inner vetos is adjusted via the precise machining of the vertical silicon standoffs (h) setting the overall height of the assembly (Color figure online)

\subsection{The Fiducial-Volume Cryogenic Detector}

The small size of the target detectors allows encapsulating them within cryogenic veto detectors. In order to reach a background rate comparable with an underground laboratory while running at shallow depth, a twofold anticoincidence veto is foreseen. Facing the target detector, a low-threshold surface veto with $4 \pi$ coverage protects against radioactive surface contaminations. In addition, it serves as an elastic detector holder providing point-like mechanical contacts to the target detectors. A second, massive cryogenic detector encapsulates the assembly of targets and inner veto. This outer veto detector functions as an anticoincidence system for double-scattering backgrounds, such as gamma and neutron backgrounds.

\section{The NUCLEUS $1 \mathrm{~g}$ Demonstrator}

To show the functionality of the cryogenic components of the NUCLEUS detector, a demonstrator was constructed using only a single cube as the target. The following sections describe ongoing developments with the different components of the NUCLEUS demonstrator. Photographs of the 1g demonstrator are shown in Fig. 1.

\subsection{Target Detectors}

NUCLEUS target detectors are currently produced three at a time, on a $20 \times 10 \times$ $5 \mathrm{~mm}^{3}$ substrate. After simultaneous production of the TES sensors, the target cubes are diced and polished. This represents a large simplification of the fabrication procedure, compared to individual production. This methodology will be scaled to large wafers for the future stages of NUCLEUS. Several hundred target cubes can be produced from a single 6-inch wafer, allowing production of a $\mathrm{kg}$-scale target with reasonable effort. 
The TES sensor is adapted from the layout used in CRESST. The TES area is scaled down in proportion with the size of the target crystal, in order to obtain the same nonthermal phonon lifetime. With a reduced tungsten area (and thus sensor heat capacity), also the thermal link strength has to be reduced to avoid speeding up the relaxation of the TES. To this end, the feature size has been reduced from 40 to $10 \mu \mathrm{m}$. In addition, studies are ongoing with a TES version using twice the tungsten area, and an unchanged CRESST-III light detector TES structure. A target cube equipped with the latter TES version achieved an energy threshold around $15 \mathrm{eV}$ in the silicon holder described below.

\subsection{Inner Veto}

The inner veto with its dual role of surface veto and instrumented holder is implemented using elastic silicon wafers of $200 \mu \mathrm{m}$ thickness, with a side length of $11 \mathrm{~mm}$. Using a wet etching process, pyramidal structures with a square tip of $100 \mu \mathrm{m}$ side length are structured into the inward surfaces of the inner veto wafers. The target is held by three pyramids on each wafer. Both inner veto wafers are equipped with a CRESST-III light detector TES on the outward surface. Additionally, the top inner veto wafer has a laser-cut window of $2 \times 2.5 \mathrm{~mm}^{2}$ to allow contacting the TES of the target cube. The demonstrator inner veto covers only the top and bottom sides of the target cube. For the physics setup, the lower plate will be replaced by a milled silicon beaker shielding also the sides of the target array, improving the geometric coverage to above $97 \%$.

In March 2019, the inner veto was operated together with a target cube in stable conditions. The three detectors in mechanical contact were calibrated using a ${ }^{55} \mathrm{Fe}$ source. The veto detectors reached baseline energy resolutions of $13.4 \mathrm{eV}$ (top) and $9.1 \mathrm{eV}$ (bottom) as determined using an optimum filter technique. While achievable trigger thresholds were not studied explicitly, a threshold below $100 \mathrm{eV}$ appears realistic. As expected, there is moderate phonon transmission across the mechanical contact between the target and the inner veto. This detector arrangement is designed to act as an instrumented holder. Pulse height ratios of 5-20\% between the target and inner vetos are observed. In the dataset (shown in Fig. 2), three event populations are clearly identifiable, corresponding to energy depositions in the three detectors. The dominant background at the above-ground, unshielded setup at the Max Planck Institute for Physics thus appears to consist of individual energy depositions (as opposed to shared energy depositions caused by back-to-back events from surface contaminations). A cut on both veto amplitudes (shown in Fig. 3) efficiently removes the event populations originating in the vetos, resulting in a flat target background spectrum down to around $200 \mathrm{eV}$. The rising background below $200 \mathrm{eV}$, i.e., in the ROI for CEvNS, is the subject of ongoing studies.

\subsection{Outer Veto}

The massive outer veto has the purpose of suppressing double interactions of external neutron and gamma backgrounds in anticoincidence with the target detectors. In the demonstrator, it is implemented by two $5 \mathrm{~cm} \times 5 \mathrm{~cm}$ silicon cylinders with a milled 

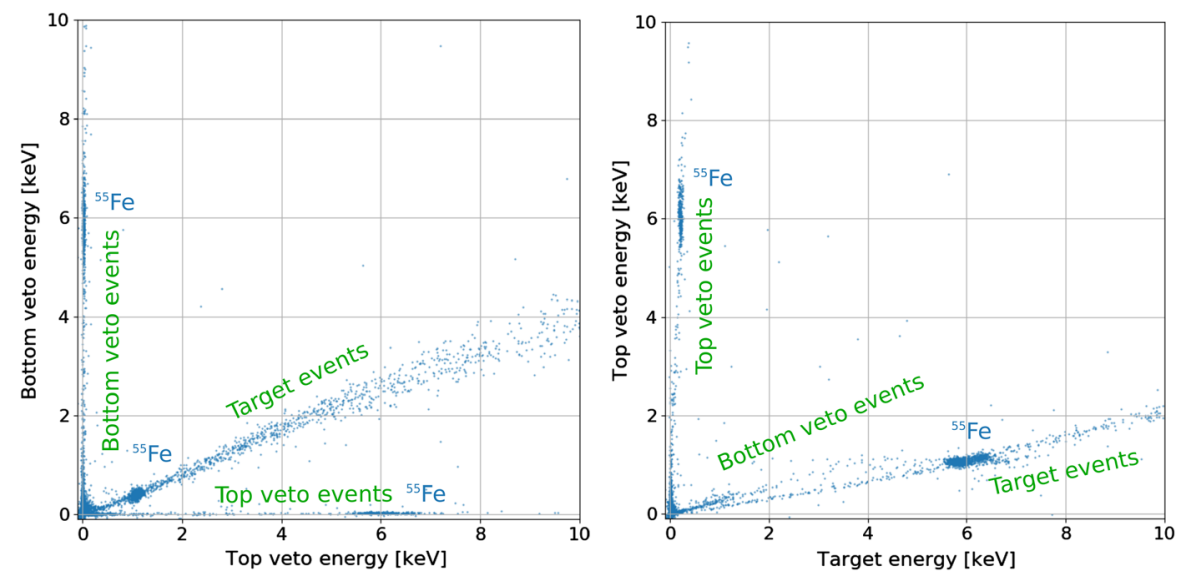

Fig. 2 Event distribution between target and inner veto detectors. Left: top veto energy versus bottom veto energy. Three bands are observed corresponding to energy depositions in the top veto, target and bottom veto. The target events appear in this plot due to phonon transmission between target and vetos through the mechanical contacts. The $6 \mathrm{keV}$ lines of the ${ }^{55} \mathrm{Fe}$ source can be identified in all three populations. Right: target energy vs. top veto energy. The bottom veto event population appears close to the target band (Color figure online)

Fig. 3 Event histogram by target energy. The blue histogram shows all events, and the orange histogram is cleaned by a cut on both veto energies (including a correction for the cut survival probability). The resulting spectrum is cleaned from energy depositions in the vetos and is flat down to $200 \mathrm{eV}$ (Color figure online)

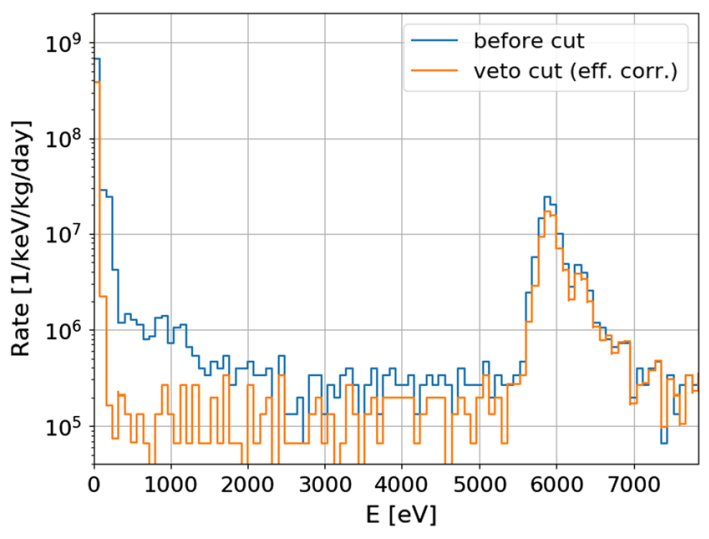

cavity for the inner detectors. Both outer veto crystals are instrumented with large-area TESs $\left(6 \times 8 \mathrm{~mm}^{2}\right)$ to achieve fast rise times in spite of the large absorber size. For a first test, the outer vetos were operated separately from the other detector components. In the unshielded setup, both detectors suffered from a large particle event rate of several tens of Hz. An energy calibration was not achieved, and further characterization is deferred to future runs in the shallow underground laboratory at the Technical University of Munich (TUM) (factor $\sim 3$ muon flux reduction, 15 m.w.e. [19]).

The $1 \mathrm{~g}$ demonstrator has proven basic functionality of all cryogenic detector systems planned for NUCLEUS. The mechanical, electrical and thermal design is validated and shown to withstand several thermal cycles. All cryogenic detectors, especially the inner ones which are in mechanical contact, can be operated with good 
performance. With these results, the design for the fiducial-volume cryogenic detector is validated.

\section{A New Experimental Site for NUCLEUS at Chooz}

In parallel with the cryogenic detector preparations, studies and measurements are ongoing to establish an experimental site for NUCLEUS in the vicinity of a nuclear power reactor and to finalize the design of the facility to be constructed there.

\subsection{The Very-Near-Site at Chooz NPP}

A new experimental site at the Chooz Nuclear Power Plant is planned to host the NUCLEUS experiment [20]. It is located between the two reactor cores with a thermal power of $4.25 \mathrm{GW}$ each, at distances of $72 \mathrm{~m}$ and $102 \mathrm{~m}$. The expected neutrino flux at the very-near-site $(\mathrm{VNS})$ is about $3 \times 10^{12} /\left(\mathrm{s} \mathrm{cm}^{2}\right)$. The VNS consists of a $24 \mathrm{~m}^{2}$ room in the basement of a five-story office building. First muon measurements performed at the VNS show an average overburden of 3 m.w.e. A measurement of the fast neutron flux at VNS and outside in the recoil energy range $100-2000 \mathrm{keV}_{\text {ee }}$ shows a reduction of the flux by a factor 8 .

\subsection{The NUCLEUS Setup at VNS}

For the experimental setup to be installed at the VNS, several components are currently in design phase. The centerpiece is a dry dilution refrigerator to be delivered to Munich early 2020. A dedicated vibration decoupling system allowing quiet detector operation is under study. Geant4 studies are ongoing to define the precise layout of a compact multi-layered shielding with a side length of 1-1.5 m. Prototypes are constructed for a plastic-scintillator muon veto covering the compact shielding. The cryostat is modified allowing for the inclusion of a cold shield covering the space above the detector array, with a mass of up to $200 \mathrm{~kg}$. The sample space for the cryogenic target is sufficient to allow future upgrades with $\mathrm{kg}$-scale total mass.

\section{Summary and Outlook}

The NUCLEUS collaboration is preparing a $10 \mathrm{~g}$ array of cryogenic detectors to measure CEvNS at Chooz NPP. The different vetos of the fiducial-volume cryogenic detector concept have been operated in a $1 \mathrm{~g}$ demonstrator. The inner veto achieved energy thresholds below $100 \mathrm{eV}$, running stably while in contact with the target detector. This shows the functionality of the inner veto as instrumented holder and surface veto. The outer veto will be characterized further in an underground setup at TUM.

The NUCLEUS collaboration is planning to commission the $10 \mathrm{~g}$ cryodetectors in Munich during 2020, leading to a "blank assembly" of all components of the experimental setup in 2021. The setup is planned to move to Chooz for commissioning in 
2021, with data taking expected to start there in early 2022. In parallel, a dedicated

R\&D effort will be undertaken toward a future upgrade of the total target mass toward $1 \mathrm{~kg}$.

Acknowledgements Open access funding provided by Max Planck Society. NUCLEUS is supported by ERC-StG-2018 "NUCLEUS," the Collaborative Research Center SFB1258 of the DFG, Excellence Cluster Universe and Excellence Cluster ORIGINS. The NUCLEUS $1 \mathrm{~g}$ demonstrator was funded by the Excellence Cluster "Universe" as a seed project in 2017 and 2018.

Open Access This article is licensed under a Creative Commons Attribution 4.0 International License, which permits use, sharing, adaptation, distribution and reproduction in any medium or format, as long as you give appropriate credit to the original author(s) and the source, provide a link to the Creative Commons licence, and indicate if changes were made. The images or other third party material in this article are included in the article's Creative Commons licence, unless indicated otherwise in a credit line to the material. If material is not included in the article's Creative Commons licence and your intended use is not permitted by statutory regulation or exceeds the permitted use, you will need to obtain permission directly from the copyright holder. To view a copy of this licence, visit http://creativecommons.org/licenses/by/4.0/.

\section{References}

1. D. Freedmann, Coherent effects of a weak neutral current. Phys. Rev. 9, 5 (1974)

2. D. Akimov et al., COHERENT Collaboration, Observation of coherent elastic neutrino-nucleus scattering. Science 357, 6356 (2017). https://doi.org/10.1126/science.aao0990

3. A. Aguilar-Arevalo et al., The CONNIE experiment. J. Phys. Conf. Ser. 761, 012057 (2016)

4. W. Maneschg, The status of CONUS, Zenodo (2018). https://doi.org/10.5281/zenodo.1286927

5. G. Agnolet, et al., MINER Collaboration, Background studies for the MINER coherent neutrino scattering reactor experiment. Nucl. Instrum. Methods Phys. Res. Sect. A Accel. 853, 53 (2017)

6. V. Belov et al., The $v$-GeN experiment at the Kalinin Nuclear Power Plant. JINST 10, P12011 (2015)

7. DYu. Akimov et al., The RED-100 two-phase emission detector. Instrum. Exp. Tech. 60, 175 (2017)

8. J. Billard et al., Coherent neutrino scattering with low temperature bolometers at Chooz reactor complex. J. Phys. G 44, 105101 (2017)

9. H.T. Wong, Neutrino-nucleus coherent scattering and dark matter searches with sub-keV germanium detector. Nucl. Phys. A 844, 229C (2010)

10. J. Barranco et al., Probing new physics with coherent neutrino scattering off nuclei. JHEP 12, 021 (2005)

11. M. Lindner et al., Coherent neutrino-nucleus scattering and new neutrino interactions. JHEP 03, 097 (2017)

12. J. Billard et al., Prospects for exploring new physics in coherent elastic neutrino-nucleus scattering. JCAP 1811, 11 (2018)

13. M. Mancuso, Searches for light dark matter with the CRESST-III experiment. JLTP. Manuscript No. JLTP-D-19-00248

14. A. H. Abdelhameed et al., CRESST Collaboration, First results from the CRESST-III low-mass dark matter program (2019) arXiv:1904.00498

15. R. Strauss et al., A prototype detector for the CRESST-III low-mass dark matter search. Nucl. Instrum. Methods A845, 414-417 (2017)

16. R. Strauss et al., Gram-scale cryogenic calorimeters for rare-event searches. Phys. Rev. D 96, 022009 (2017)

17. R. Strauss et al., The $v$-cleus experiment: a gram-scale fiducial-volume cryogenic detector for the first detection of coherent neutrino-nucleus scattering. Eur. Phys. J. C 77, 506 (2017). https://doi.org/10. 1140/epjc/s10052-017-5068-2

18. G. Angloher et al., Results on MeV-scale dark matter from a gram-scale cryogenic calorimeter operated above ground. Eur. Phys. J. C 77, 637 (2017)

19. A. Langenkämper et al., A cryogenic detector characterization facility in the shallow underground laboratory at the Technical University of Munich. J. Low Temp. Phys. 193, 860-866 (2018). https:// doi.org/10.1007/s10909-018-1899-y 
20. G. Angloher et al., Exploring CEvNS with NUCLEUS at the Chooz Nuclear Power Plant. arXiv: 1905.10258

Publisher's Note Springer Nature remains neutral with regard to jurisdictional claims in published maps and institutional affiliations.

\section{Affiliations}

J. Rothe ${ }^{1}(1) \cdot G$. Angloher ${ }^{1} \cdot$ F. Ardellier-Desages ${ }^{2,3} \cdot$ A. Bento ${ }^{1,4}$.

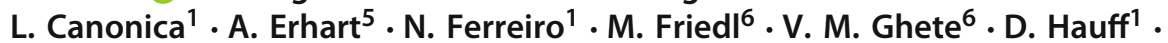

H. Kluck ${ }^{6,7}$ - T. Lasserre ${ }^{2,3}$. D. Lhuillier ${ }^{2}$. A. Kinast ${ }^{5} \cdot$ M. Mancuso $^{1}$.

J. Molina Rubiales ${ }^{8}$. E. Mondragon-Cortes ${ }^{5}$. G. Munch ${ }^{8}$. C. Nones ${ }^{2}$.

L. Oberauer ${ }^{5}$. T. Ortmann ${ }^{5} \cdot$ L. Pattavina ${ }^{5} \cdot$ F. Petricca ${ }^{1} \cdot$ W. Potzel ${ }^{5}$.

F. Pröbst ${ }^{1} \cdot$ F. Reindl ${ }^{6,7}$. J. Schieck ${ }^{6,7}$. S. Schönert ${ }^{5} \cdot$ C. Schwertner ${ }^{6,7}$.

L. Scola ${ }^{2} \cdot$ L. Stodolsky ${ }^{1} \cdot$ R. Strauss ${ }^{5} \cdot$ M. Vivier ${ }^{2} \cdot$ V. Wagner ${ }^{2}$.

A. Zolotarova ${ }^{2}$. The NUCLEUS Collaboration

$凶 \quad$ J. Rothe

jrothe@mpp.mpg.de

1 Max-Planck-Institut für Physik, 80805 München, Germany

2 IRFU, CEA, Université Paris Saclay, 91191 Gif-sur-Yvette, France

3 APC, Astro Particule et Cosmologie, Université Paris Diderot, CNRS/IN2P3, CEA/Irfu, Observatoire de Paris, Sorbonne Paris Cité, 10, rue Alice Domon et Leonie Duquet, 75205 Paris Cedex 13, France

4 CIUC, Departamento de Fisica, Universidade de Coimbra, 3004516 Coimbra, Portugal

5 Physik-Department, Technische Universität München, 85748 Garching, Germany

6 Institut für Hochenergiephysik der Österreichischen Akademie der Wissenschaften, 1050 Wien, Austria

7 Atominstitut, Technische Universität Wien, 1020 Wien, Austria

8 Électricité de France, Centre nucléaire de production d'électricité de Chooz, Service Automatismes-Essais, 08600 Givet, France 